\title{
A Mechanism to Explain Ototoxicity in Neonates Exposed to Bumetamide: Lessons to Help Improve Future Product Development in Neonates
}

\author{
Karel Allegaert ${ }^{1,2}\left(\mathbb{D} \cdot\right.$ Amir Lahav $^{3} \cdot$ John N. van den Anker ${ }^{1,4,5}$
}

Published online: 18 August 2016

(c) Springer International Publishing Switzerland 2016

\section{Introduction}

The most prominent feature of neonatal clinical pharmacology is huge variability in pharmacokinetics and pharmacodynamics of frequently used medicines due to rapid growth and maturation. This variability is further aggravated by other covariates such as disease characteristics, co-medication or pharmacogenetics. A thorough understanding of developmental changes affecting pharmacokinetics (absorption, distribution, metabolism, elimination) in neonates is essential to provide accurate dose adjustments. While there is increasing knowledge on the impact of these maturational changes on pharmacokinetics, there is currently very limited data on developmental pharmacodynamics [1]. The fact that adverse drug events in neonates are common and may have lifelong consequences, focused pharmacovigilance is urgently needed. Even more, pharmacovigilance should stimulate us to try unveiling the

Karel Allegaert

karel.allegaert@uzleuven.be

1 Intensive Care and Department of Surgery, Erasmus MC-Sophia Children's Hospital, Rotterdam, The Netherlands

2 Department of Development and Regeneration, KU Leuven, Leuven, Belgium

3 Department of Pediatrics, Mass General Hospital for Children, Harvard Medical School, Boston, MA 02114, USA

4 Division of Paediatric Pharmacology and Pharmacometrics, University of Basel Children's Hospital, Basel, Switzerland

5 Division of Clinical Pharmacology, Children's National Health System, Washington, DC, USA underlying mechanisms of adverse events, and to use this newly acquired knowledge in redesigning the product development approach in neonates.

\section{A Mechanism to Explain Ototoxicity in Neonates Participating in the NEMO Trial}

The urgent need for a pharmacovigilance program in neonates specifically focusing on ototoxicity has recently been highlighted in the NEMO (treatment of NEonatal seizures with Medication Off-patent) trial. The aim of this study was to treat resistant neonatal seizures in asphyxia with different, relatively high, doses of bumetanide $(0.05-0.3 \mathrm{mg} / \mathrm{kg}$ every $12 \mathrm{~h})$ after a loading dose of phenobarbital. This clinical trial was stopped early because of lack of efficacy and a high incidence of serious adverse events, including severe hearing impairments in three out of 11 survivors, unrelated to measured trough concentrations of aminoglycosides [2].

We would like to propose a pharmacological mechanism that might explain why neonates, who are treated with bumetanide, are more prone to develop ototoxicity and to propose considering animal studies that will assess hearing related safety aspects in addition to pharmacovigilance.

In vitro studies have shown that aminoglycosides bind tightly to acidic phospholipids and alter intracellular trafficking. While this binding may potentially occur at any cell, it is much more pronounced at cochlear hair cells due to expression of specific ligands (like megalin and the colocalized cubilin) at the brush border of the cells in the inner ear. The subsequent process of endocytosis also 
involves megalin as multiligand endocytic receptor. Intracellular accumulation subsequently will induce processes that may result in cell death through apoptosis. Besides endocytosis, there are also endocytosis-independent pathways using cation channels [3]. Interestingly, in vitro models have documented that loop diuretics like furosemide or bumetanide enhance aminoglycoside uptake through hyperpolarization of the cell, increasing cation influx cation force [4]. It is therefore reasonable to hypothesize that in the NEMO trial the pharmacodynamic effect of bumetanide stimulated a cation influx force that resulted in intracellular aminoglycoside accumulation, subsequent toxicity, and unavoidable cell death [4]. In addition, based on animal studies, the mechanism of megalin-independent ion channel driven accumulation of aminoglycosides has also been suggested to explain the synergism between exposure to noise and aminoglycoside toxicity [5]. Exposure to loud noise also increases the probability of a current through mechano-electrical transduction using ion channels which-in turn-will result in a higher intracellular aminoglycoside accumulation within the hair cells, subsequent cell death, and ultimately hearing loss.

The outcome of the NEMO trial is unfortunate but is in line with already available observations on furosemide related hearing impairments [6]. While the aforementioned mechanism has only been reported recently, the epidemiological data between loop diuretics and hearing impairment have been reported decades ago, using much lower doses of bumetanide. In retrospect, this association could have been considered and assessed more rigorously in a maturational animal model before conducting clinical trials in humans.

\section{How Should This Help us to Improve Product Development in Neonates?}

Neonates are still in an urgent need for safer and more effective drugs for their diseases. This is also true for neonatal seizures because this is a very relevant disease entity which deserves better clinical management and drug choices [1]. However, effective product development in neonates necessitates multidisciplinary collaboration, and we claim that is up to us-clinicians- to reach out to other disciplines trying to stimulate them to build preclinical models tailored to explore the mechanism of disease entities and compounds to treat these entities (e.g., maturational ototoxicity [5]) in neonates.

Decisions on compound, indication, timing and dosing for a clinical trial in human neonates should be based on a multidisciplinary team decision (including developmental toxicologists, clinical pharmacologists, and trial specialists) and must take into account: (a) clinical needs, (b) available epidemiological data, and (c) evidence supported by juvenile animal studies in assuring sufficient efficacy and safety.

The good news is that these kinds of multidisciplinary collaborations with a focus on neonatal drug development are currently emerging. As part of the Critical Path Initiative (C-path), the International Neonatal Consortium (INC) has recently been developed to serve as a multidisciplinary forum for the neonatal community to develop consensus statements (e.g., standardization of methods, standard of care consensus statements, population specific biomarkers, modelling approaches, trial designs, clinical outcome assessment tools, formulation issues) with the ultimate goal to improve neonatal medicines development [7]. Similarly, the Developmental and Reproductive Toxicology (DART) technical committee as part of the Health and Environmental Sciences Institute (HESI) scientific initiative has launched a project to identify the available non-clinical models to facilitate the optimal use of nonclinical approaches for neonatal therapeutics. This initiative aims to collect and report on an extensive survey on nonclinical models of neonatal pediatric therapeutics. In the same effort, this initiative also aims to establish a framework for evaluation and implementation of nonclinical neonatal models, to improve the understanding of comparative, inter-species maturation, and to optimize dose selection in neonates $[8,9]$.

At least multidisciplinary approaches from pre-clinical to clinical studies in neonates may also reshape our approach to medicine development in neonates, from exploratory approaches to focused product development driven by their needs (diseases), characteristics (maturational physiology) and tolerance (maturational toxicology).

Acknowledgments K Allegaert has been supported by the Fund for Scientific Research, Flanders (fundamental clinical investigatorship $1800214 \mathrm{~N}$ ). The research activities are further facilitated by the agency for innovation by Science and Technology in Flanders (IWT) through the SAFEPEDRUG project (IWT/SBO 130033). J van den Anker has been supported by the Eunice Kennedy Shriver National Institute of Child Health and Human Development (U54HD071601, T32HD087969).

Compliance with ethical standards K Allegaert, A Lahav and J van den Anker have no conflicts of interest to report. Besides the entities mentioned in the acknowledgements section, there were no other sources of funding used to support the writing of this manuscript.

\section{References}

1. Allegaert K, van den Anker J. Neonatal drug therapy: the first frontier of therapeutics for children. Clin Pharmacol Ther. 2015;98:288-97.

2. Pressler RM, Boylan GB, Marlow N, Blennow M, Chiron C, Cross $\mathrm{JH}$, et al. Bumetanide for the treatment of seizures in newborn 
babies with hypoxic ischaemic encephalopathy (NEMO): an openlabel, dose finding, and feasibility phase $1 / 2$ trial. Lancet Neurol. 2015;14:467-77.

3. Nagai J, Takano M. Entry of aminoglycosides into renal tubular epithelial cells via endocytosis-dependent and endocytosis-independent pathways. Biochem Pharmacol. 2014;90:331-7.

4. Wang T, Yang YQ, Karasawa T, Wang Q, Phillips A, Guan BC, et al. Bumetanide hyperpolarizes madin-darby canine kidney cells and enhances cellular gentamicin uptake by elevating cytosolic $\mathrm{Ca}(2+)$ thus facilitating intermediate conductance $\mathrm{Ca}(2+)$-activated potassium channels. Cell Biochem Biophys. 2013;65:381-98.

5. Zimmerman E, Lahav A. Ototoxicity in preterm infants: effects of genetics, aminoglycosides, and loud environmental noise. J Perinatol. 2013;33:3-8.
6. Pacifici GM. Clinical pharmacology of furosemide in neonates: a review. Pharmaceuticals. 2013;6:1094-129.

7. International Neonatal Consortium. Accelerating the development of safe and effective therapies for neonates. Critical Path Institute. http://c-path.org/programs/inc. Accessed 20 July 2016.

8. The teratology society, births defect research association. http:// connection.teratology.org/p/bl/et/blogid=4\&blogaid=660. Accessed 20 July 2016.

9. ILSI Health and Environmental Sciences Institute. http://hesiglobal. org/wp-content/uploads/sites/11/2016/06/DART-Committee-FactSheet.pdf. Accessed 20 July 2016. 